\title{
Quadcopter Trajectory Tracking Control using State-Feedback Control with Integral Action
}

\author{
Ahmed Samir \\ Faculty of Engineering at Shoubra, Benha University, Egypt \\ 108 Shoubra, Cairo Governorate, Egypt \\ Ashraf Hafez \\ Faculty of Engineering at Shoubra, Benha University, Egypt \\ 108 Shoubra, Cairo Governorate, Egypt
}

\author{
Abdallah Hammad \\ Faculty of Engineering at Shoubra, Benha University, Egypt \\ 108 Shoubra, Cairo Governorate, Egypt \\ Hala Mansour \\ Faculty of Engineering at Shoubra, Benha University, Egypt \\ 108 Shoubra, Cairo Governorate, Egypt
}

\begin{abstract}
Trajectory tracking of Unmanned Aerial Vehicles (UAVs) is a very challenging and complicated field of research due to their nonlinear and underactuacted dynamics. In this paper, a real time trajectory tracking controller is developed for a quadrotor. A state feedback with integral action control scheme is designed for the position controller to ensure that the quadrotor can track the reference position rapidly. The quadrotor dynamic model is established using Newton-Euler formalism taking into account various physical phenomena that can effect its dynamic behavior. NI-LabVIEW based simulation results show that the proposed controller can make the quadrotor tracks the desired trajectory quickly and smoothly with ensuring the stability for roll and pitch angle.
\end{abstract}

\section{Keywords}

Quadcopter, Realtime, Tracking, Navigation, Trajectory, Modling, State feedback

\section{INTRODUCTION}

More recently, quadrotor UAV becomes very popular. Taken broadly, it is a sort of new kind of unmanned aerial vehicle. But compared to the traditional UAV, quadrotor is simpler, smaller, and has lower cost. It can not only fly in three-dimensional space flexibly, but also can gather information through on-board sensors. Due to that, it has been broadly utilized in several field, such as rescue missions, aerial photography, investigation, and mapping especially in narrow space and more risky environment [1]-[3].

Quadrotor has six degrees of freedom in space, but because of the restriction of structure, it can only be using four independent motors, so the quadrotor is a sort of typical under-actuated system. The flight mechanism is as the following: vertical movement is made by collectively increasing and decreasing the equally speed of every rotor. Pitch movement is accomplished by the differential speed of the front back set of rotors. Roll is accomplished by the differential speed of the left right set of rotors. Yaw motion is realized by the different reactive torques [4].

As specified, many common control algorithms for UAV are not applicable for quadrotor due to its under-actuation. So late years, control methodology and engineering application for quadrotor has gotten more consideration of scholars. These kinds of UAVs have a high nonlinear and time-varying behavior, parametric uncertainties and are affected by atmospheric turbulence. Therefore, a robust adaptive controller is required to achieve accurate tracking and high performances in an autonomous flight with disturbance rejection capabilities [5].

The problem of trajectory tracking has been proposed of several controller. Many traditional control techniques have been applied such as PD control[6]. PID control [7][8] and LQR control method [8].Nevertheless, PD and PID control algorithm were used to understand the tracking control of quadrotor in [6][7]. To reduce the steady state error, the gain of PID must be increased but the system was easily affected by outer disturbance.

The underactuated trajectory tracking problem was handled using backstepping in [9] and sliding mode control techniques in [10][11]. In [12] the authors gave a solution to the time scale separation assumption by means of a tracking differentiator and used a new command filtered compensation for quadrotor trajectory control. Nested saturation algorithm combined with backstepping and feedback control technique has been used in [13].

The authors in [14] proposed a nonlinear adaptive tracking controller based on backstepping techniques that can estimate and compensate the effect of mass uncertainty. In [15] a state feedback control system and an integrator backstepping approach was used for tracking control. This paper presents implementation of a real time trajectory tracking controller using state feedback control with integral part.

This paper is organized as follows: The current section is introduction. Section 2 presents a description of the basic structure, and dynamic model of quadrotor. The state feedback controller with integral part architecture is introduced in section 3 Section 4 presents simulation results to validate the proposed controller. The conclusions and the future work are given in section 5

\section{DYNAMIC MODEL OF QUADROTOR}

The quadcopter depicted in Fig. 1 consists of a six degree offreedom(DOF) body actuated by four propellers. The four rotors provide upwards thrusting as well direction control. The motors are located at the four corners of the quadcopter. The motors situated diagonally opposite to each other rotate in the same 
direction while the adjacent motors rotate in the counter direction. The mathematical model was developed using Newton-Euler dy-

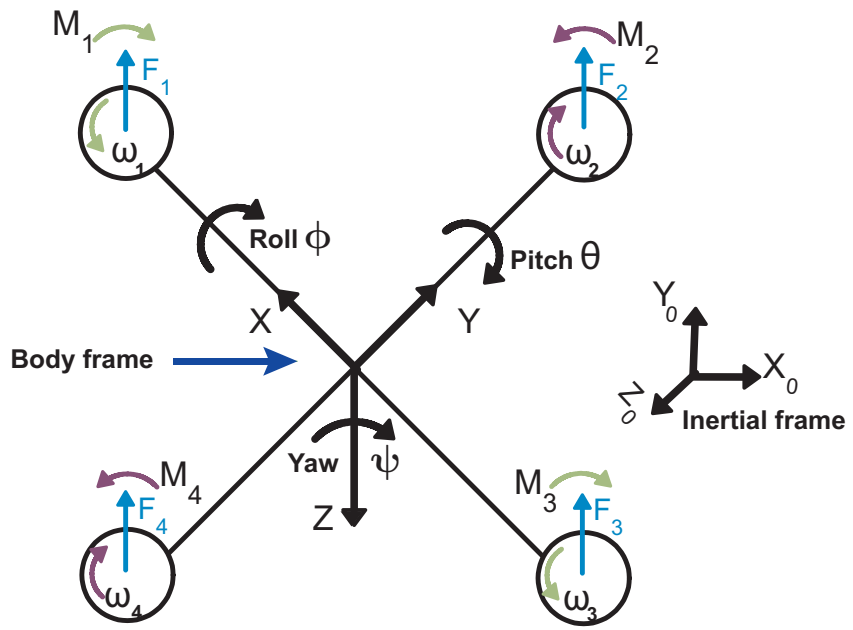

Fig. 1. A typical structure of quadrotor

namic equations using the following simplifying assumptions [16] :

- The structure including the motors are rigid.

- The gyroscopic effects of the propellers are negligible.

- The quadcopter has two planes of symmetry, i.e., the products of inertia are equal to zero, $I x y=I y z=I x z$.

The mathematical model are given as follows :

$$
\begin{gathered}
\ddot{\phi}=\frac{L}{I_{x x}} U_{2}-\frac{J_{r}}{I_{x x}} \omega_{r} \dot{\theta}+\frac{I_{y y}-I_{z z}}{I_{x x}} \dot{\psi} \dot{\theta}, \\
\ddot{\theta}=\frac{L}{I_{y y}} U_{3}-\frac{J_{r}}{I_{y y}} \omega_{r} \dot{\phi}+\frac{I_{z z}-I_{x x}}{I_{y y}} \dot{\psi} \dot{\phi}, \\
\ddot{\psi}=\frac{1}{I_{z z}} U_{4}+\frac{I_{x x}-I_{y y}}{I_{z z}} \dot{\phi} \dot{\theta}, \\
\ddot{x}=\frac{-U_{1}}{m}(\sin \phi \sin \psi+\cos \phi \cos \psi \sin \theta) \\
\ddot{y}=\frac{-U_{1}}{m}(\cos \phi \sin \psi \sin \theta-\sin \phi \cos \psi), \\
\ddot{z}=g-\frac{U_{1}}{m}(\cos \phi \cos \theta) .
\end{gathered}
$$

where the parameters using in eq. (1) - (6) are described in table (1).

The system has six outputs $(x, y, z, \phi, \theta, \psi)$ and four inputs $\left(U_{1}, U_{2}\right.$, $\left.U_{3}, U_{4}\right)$. The control input $U_{1}$ provides thrust on the body in the z-axis, $U_{2}$ and $U_{3}$ are the roll and pitch inputs and $U_{4}$ is used for yaw control. The control inputs $U_{i}$ can be written in terms of rotor
Table 1. Variable description

\begin{tabular}{||c|c|c||}
\hline \hline Parameter & Unit & Descritpion \\
\hline$\phi$ & $\mathrm{rad}$ & Roll angle \\
$\theta$ & $\mathrm{rad}$ & Pitch angle \\
$\psi$ & $\mathrm{rad}$ & Yaw angle \\
$\omega_{r}$ & $\mathrm{rad} / \mathrm{sec}$ & Angular velocity of the propeller blades \\
$J_{r}$ & $\mathrm{~kg} \cdot \mathrm{m}^{2}$ & Moment of inertia of the propeller blades \\
$I_{x x}, I_{y y}, I_{z z}$ & $\mathrm{~kg} \cdot \mathrm{m}^{2}$ & Moments of inertia of the quadcopter \\
$L$ & $\mathrm{~m}$ & Length from the rotors to the center of mass \\
$\mathrm{m}$ & $\mathrm{kg}$ & Mass of the quadrotor \\
$U_{i}$ & $\mathrm{~N}$ & Control inputs \\
$K_{F}$ & $\mathrm{~N} . \mathrm{s}^{2}$ & Aerodynamic force coefficient \\
$K_{M}$ & $\mathrm{~N} \cdot \mathrm{m} . \mathrm{s}^{2}$ & Aerodynamic moment coefficient \\
\hline \hline
\end{tabular}

speeds $\omega_{r}$ as:

$$
U=\left\{\begin{array}{l}
U_{1}=-k_{f}\left(\omega_{1}^{2}+\omega_{2}^{2}+\omega_{3}^{2}+\omega_{4}^{2}\right) \\
U 2=k_{f} L\left(-\omega_{2}^{2}+\omega_{4}^{2}\right) \\
U 3=k_{f} L\left(\omega_{1}^{2}-\omega_{3}^{2}\right) \\
U_{4}=k_{M} L\left(\omega_{1}^{2}-\omega_{2}^{2}+\omega_{3}^{2}-\omega_{4}^{2}\right)
\end{array}\right.
$$

To transform the coordinates from body-fixed frame of quadcopter to Earth fixed frame the rotation matrix $R$ is used and is given below:

$$
R=\left(\begin{array}{ccc}
C_{\theta} C_{\psi} & S_{\phi} S_{\theta} C_{\psi}-C_{\phi} S_{\psi} & C_{\phi} S_{\theta} C_{\psi}+S_{\phi} S_{\psi} \\
C_{\phi} S_{\psi} & S_{\phi} S_{\theta} S_{\psi}+C_{\phi} C_{\psi} & C_{\phi} S_{\theta} S_{\psi}-S_{\phi} C_{\psi} \\
-S_{\theta} & S_{\phi} C_{\theta} & C_{\phi} C_{\theta}
\end{array}\right)
$$

where $C$ and $S$ denote $\cos$ and $\sin$ respectively.

\section{QUADCOPTER CONTROLLER DESIGN}

State feedback control [21] is a strategy utilized in feedback control system theory to put the closed-loop poles of a plant in pre-decided areas in the s-plane. Placing poles is desirable in light of the fact that the area of the poles relates straightforwardly to the eigenvalues of the system, which control the qualities of the reaction of the system. This method is broadly utilized as a part of system with multiple inputs and multiple outputs. Transfer function can be represented by the state space equation:

$$
\dot{x}=A x+B U,
$$

with output equation :

$$
y=C x+D U,
$$

then the poles of the system are the roots of the characteristic equation given by :

$$
|s I-A|=0,
$$

state feedback is used by dominant the input vector $u$ :

$$
U=-K_{0} x,
$$

substituting into eq. 9] and (10) :

$$
\begin{aligned}
& \dot{x}=\left(A-B K_{0}\right) x \\
& y=\left(C-D K_{0}\right) x
\end{aligned}
$$

the feedback matrix $K_{0}$ which drive the closed-loop eigenvalues to the pole position fixed by the required characteristic equation. 


\subsection{State Feedback with Integral Control}

Originally, integral action was utilized in controller design to minimize steady state errors. Much of the time, due to in precision of linearization of quad model, the controller gains cann't be adjusted precise and there will be a steady state error. The solution is to guarantee an integral part in the controller design. Fig. 2] shows controller block diagram.

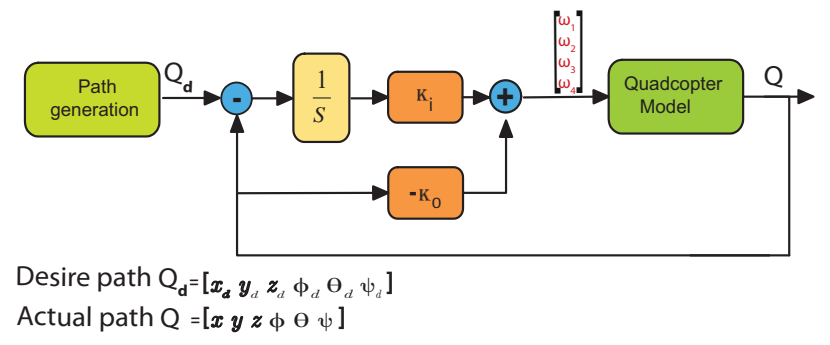

Fig. 2. Controller block diagram

\section{SIMULATION RESULTS}

The simulation results are obtained by using NI-LabVIEW. Simulations were carried out using several scenarios in order to verify the performance of the proposed controller for the trajectory tracking problem. The values of the model parameters used in the simulations are shown in Table 2 The controller parameters are chosen as : integral gain matrix,

$$
K_{i}=\left[\begin{array}{cccccc}
0 & 0 & 0 & 0.4 & 0 & 0.35 \\
0 & 0 & 0 & 0.4 & 0 & -0.35 \\
0 & 0 & 0 & 0.4 & 0.35 & 0 \\
0 & 0 & 0 & 0.4 & -0.35 & 0
\end{array}\right]
$$

and state feedback matrix,

$$
K_{0}=\left[\begin{array}{cccccc}
-0.4 & -10^{-4} & 67.7 & 3.9 & -10^{-5} & 1.4 \\
-0.4 & -10^{-4} & -67.7 & 3.9 & -10^{-5} & -1.4 \\
0.4 & -67.6 & -10^{-4} & 3.9 & 1.4 & 10^{-5} \\
0.4 & 67.6 & -10^{-4} & 3.9 & -1.4 & -10^{-5}
\end{array}\right]
$$

The sampling rate is $100 \mathrm{~Hz}$.

Table 2. Quadrotor model parameters

\begin{tabular}{||c|c|c|c||}
\hline \hline Symbol & Definition & value & Unit \\
\hline$m$ & Mass & 1.3 & $\mathrm{Kg}$ \\
$g$ & Local gravity & 9.8 & $\mathrm{~m} / \mathrm{s}^{2}$ \\
$k_{f}$ & Aerodynamic force Constant & $1.08 \mathrm{E}-06$ & $\mathrm{~N} . \mathrm{s}^{2}$ \\
$k_{m}$ & Aerodynamic moment Constant & $1.08 \mathrm{E}-08$ & $\mathrm{~N} . \mathrm{m} \cdot \mathrm{s}^{2}$ \\
$I_{x x}$ & Moment of inertia about x & 0.128 & $\mathrm{~kg} \cdot \mathrm{m}^{2}$ \\
$I_{y y}$ & Moment of inertia about y & 0.128 & $\mathrm{~kg} \cdot \mathrm{m}^{2}$ \\
$I_{z z}$ & Moment of inertia about z & 0.256 & $\mathrm{~kg} \cdot \mathrm{m}^{2}$ \\
$L$ & Moment arm & 0.21 & $\mathrm{~m}$ \\
\hline \hline
\end{tabular}

\subsection{Way-Points Tracking :}

The position of the next way-point is sent to position controller which directs the UAV towards the goal. Fig. 344 show a square trajectory defined by four way-points that described in table 3 The task was climbing to $2 \mathrm{~m}$ from the ground and follow the four waypoints of a square of $5 \mathrm{~m}$ side. In order to track the square trajectory, the controller takes the $\left(x_{d}, y_{d}\right)$ position references, and consequently the position controller produces the $\left(\theta_{d}, \phi_{d}\right)$ attitude references. Fig. 3.(a),(b) show the tracking response in $\mathrm{y}$ and $\mathrm{x}$ directions. Fig. 3 (c),(d) show the attitude response. Fig. 4 (a) shows the altitude response. Fig. 4(b) shows the yaw response. Fig. 4 (c),(d) show the tracking performance of the quad in 2d-plan and 3d-plan.
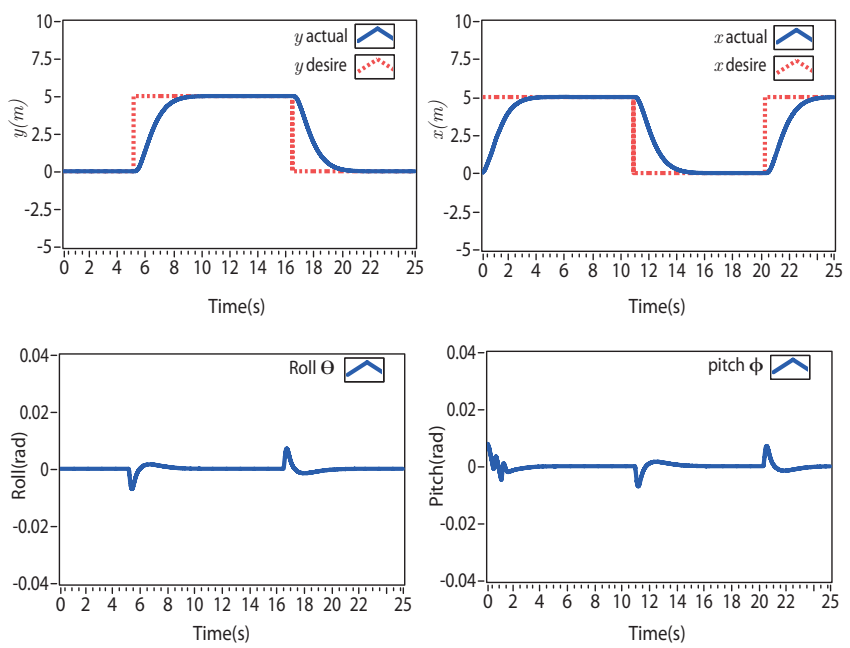

Fig. 3. (a) The desired and the actual values of y. (b) The desired and the actual values of $\mathrm{x}$. (c) the roll response. (d) the pitch response.

Table 3. Way points

\begin{tabular}{||c|c|c||}
\hline start & & $(0,0,0)$ \\
\hline Point1 & P1 & $(5,0,2)$ \\
Point2 & P2 & $(5,5,2)$ \\
Point3 & P3 & $(0,5,2)$ \\
Point4 & P4 & $(0,0,2)$ \\
\hline
\end{tabular}

Table 4 shows a summary of the performance of the system in terms of its settling time and overshoot.

Table 4. Controller Response Results

\begin{tabular}{||c|c|c|c||}
\hline \hline & Desired Value & Settling Time & Overshoot \\
\hline Altitude $(z)$ & $2 \mathrm{~m}$ & $1.4 \mathrm{sec}$ & $1.40 \%$ \\
\hline Attitude $(\theta) \operatorname{and}(\phi)$ & 2 & $0.5 \mathrm{sec}$ & $2 \%$ \\
\hline Position $(x) \operatorname{and}(y)$ & $5 \mathrm{~m}$ & $4 \mathrm{sec}$ & $0 \%$ \\
\hline \hline
\end{tabular}



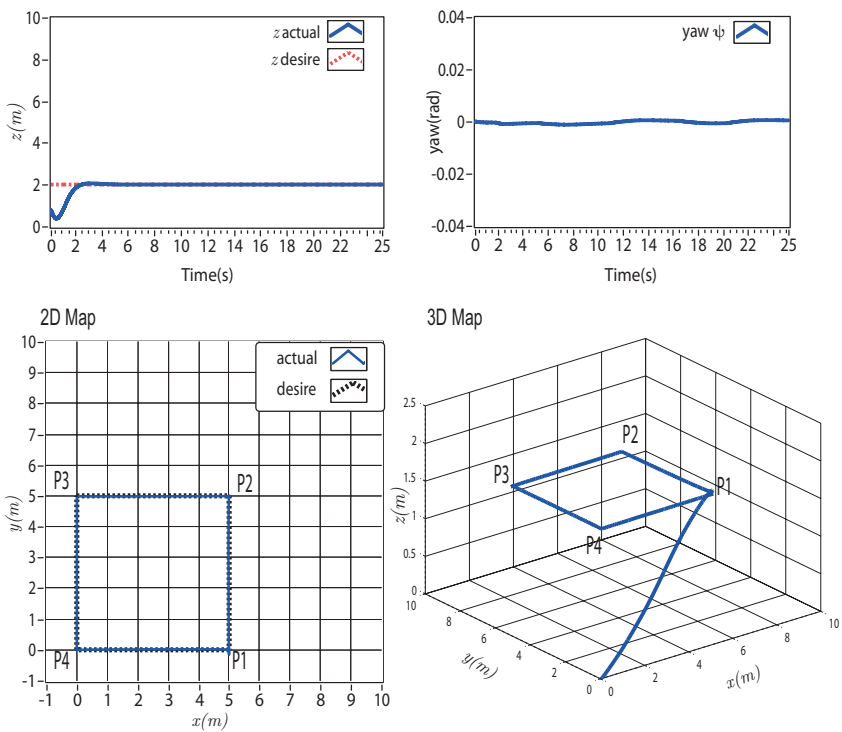

Fig. 4. Simulation result for tracking multi-point. (a) the altitude response. (b) the yaw response. (c) The tracking performance in $2 \mathrm{~d}$. (d) The tracking performance in $3 \mathrm{~d}$.

\subsection{Line Path Tracking :}

Fig. 577 show a line trajectory tracking defined by two points (start point and end point). The task was climbing to $2 \mathrm{~m}$ from the ground $(0,0,0)$ and follow the line between point $(0,0,2) \mathrm{m}$ to point $(5,5,2) \mathrm{m}$. Fig. 5(a),(b) show the tracking response in $y$ and $x$ directions. Fig. 6 (a),(b) show the attitude response. Fig. 7(a) shows the altitude response. Fig. 7(b) shows the yaw response. Fig. 7)(c),(d) show the line tracking performance of the quad in 2d-plan and 3d-plan.
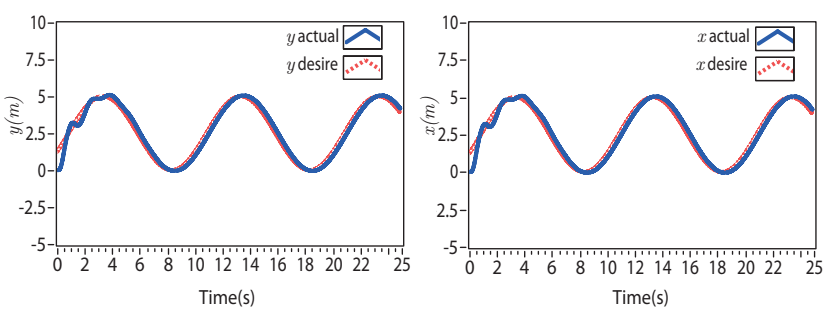

Fig. 5. (a) The desired and the actual values of $y$. (b) The desired and the actual values of $x$.
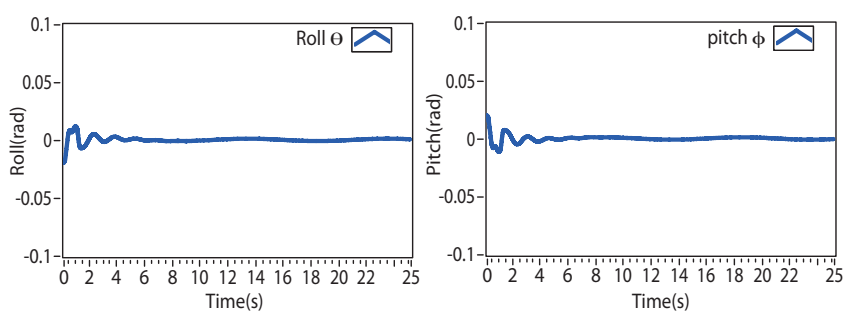

Fig. 6. (a) the roll response. (b) the pitch response.
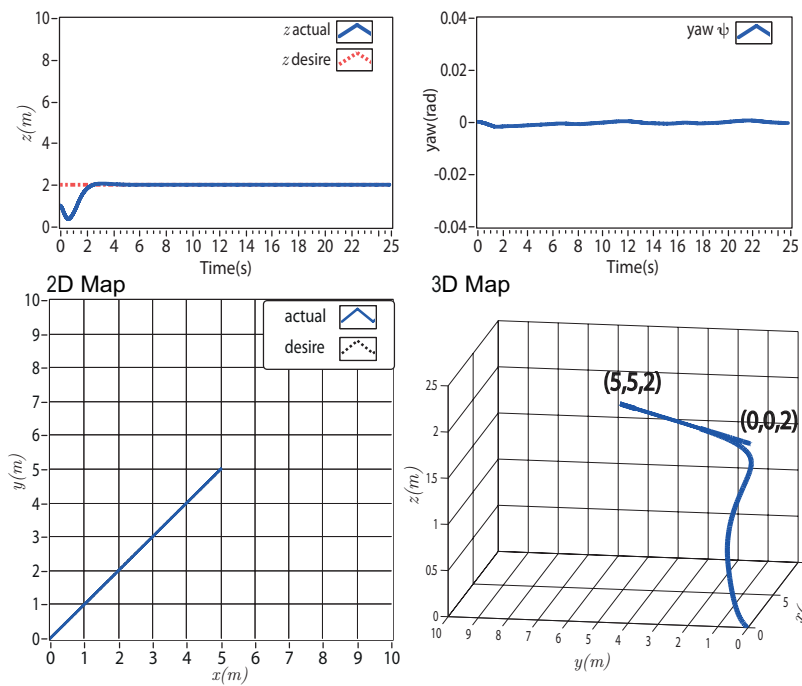

3D Map

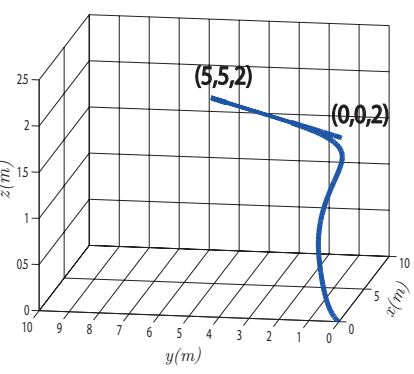

Fig. 7. Simulation result of line tracking. (a) the altitude response. (b) the yaw response. (c) The tracking performance in $2 d$. (d) The tracking performance in $3 \mathrm{~d}$.

\subsection{Circular Path Tracking :}

The task was climbing to $2 \mathrm{~m}$ from the ground and follow the a circle of center $(3,3) \mathrm{m}$ and radius $(3 \mathrm{~m})$. the path described as $x_{d}(t)=3+3 \sin 0.2 \pi t$ and $y_{d}(t)=3-3 \cos 0.2 \pi t$, or in general $\left(x_{d}-3\right)^{2}+\left(y_{d}-3\right)^{2}=9$. Fig. 8(a),(b) show the tracking response in $\mathrm{y}$ and $\mathrm{x}$ directions. Fig. 9. (a),(b) show the attitude response. Fig. 10. (a) shows the altitude response. Fig. 10 (b) shows the yaw response. Fig. 11.(a),(b) show the circular tracking performance of the quad in 2d-plan and 3d-plan.
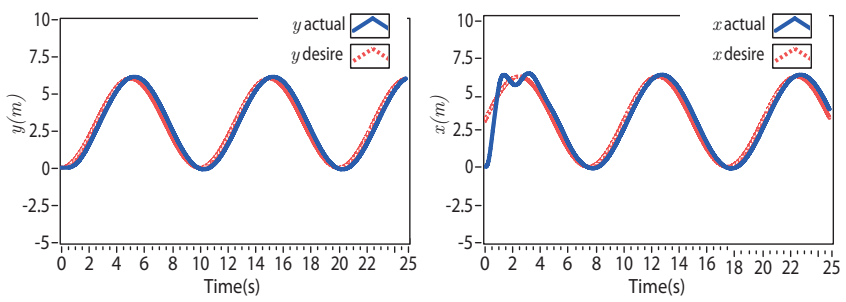

Fig. 8. The position signals generated to track the circle trajectory. (a) The desired and the actual values of $y$. (b) The desired and the actual values of $\mathrm{X}$.
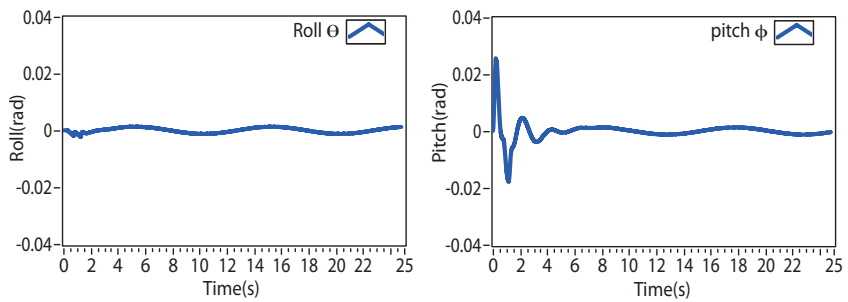

Fig. 9. The attitude signals generated to track the circle trajectory (a) the roll response. (b) the pitch response. 

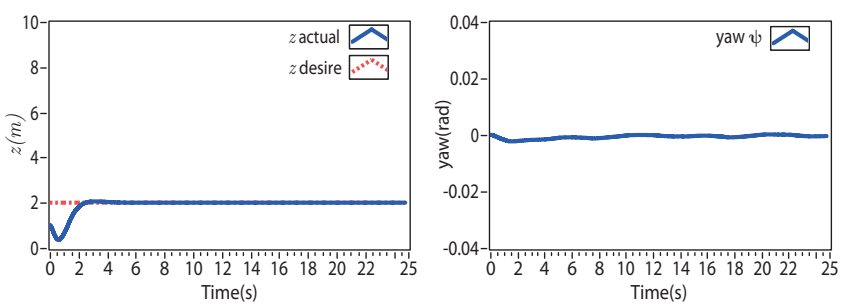

Fig. 10. (a) the altitude response. (b) the yaw response.
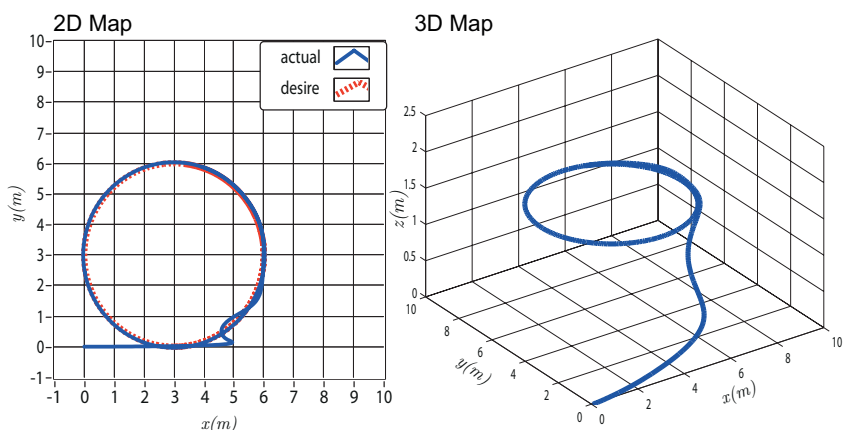

Fig. 11. Simulation result of circular path tracking. (a) The tracking performance in $2 \mathrm{~d}$. (b) The tracking performance in $3 \mathrm{~d}$.

\subsection{Ellipsoidal Path Tracking :}

The task was climbing to $2 \mathrm{~m}$ from the ground and follow the a ellipse. The path described as $x_{d}(t)=3+3 \sin 0.2 \pi t$ and $y_{d}(t)=$ $3-3 \cos (0.2 \pi t+\pi / 4)$. Fig. 12 (a),(b) show the tracking response in $y$ and $x$ directions. Fig. 13 (a),(b) show the attitude response. Fig. 14 (a) shows the altitude response. Fig. 14 (b) shows the yaw response. Fig. 15 (a),(b) show the ellipse tracking performance of the quad in 2d-plan and 3d-plan.
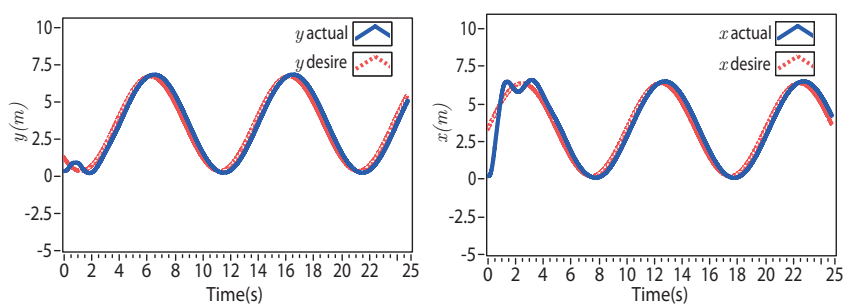

Fig. 12. The position signals generated to track the ellipse trajectory. (a) The desired and the actual values of $y$. (b) The desired and the actual values of $\mathrm{x}$.
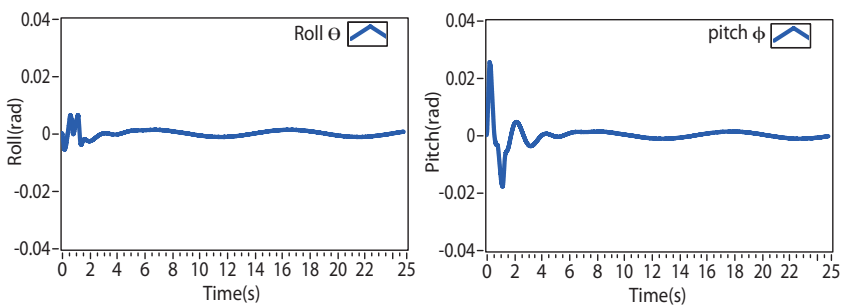

Fig. 13. The attitude signals generated to track the ellipse trajectory. (a) the roll response. (b) the pitch response.
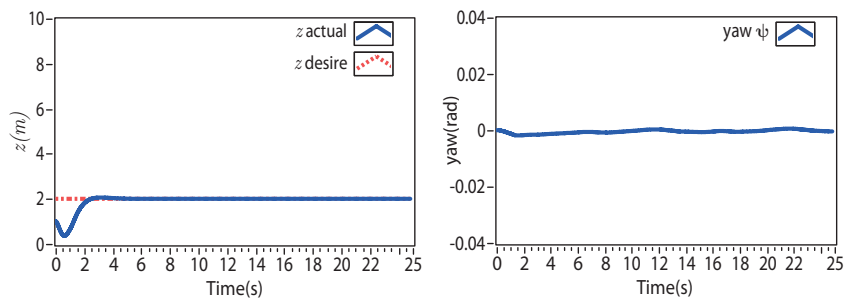

Fig. 14. (a) the altitude response. (b) the yaw response.
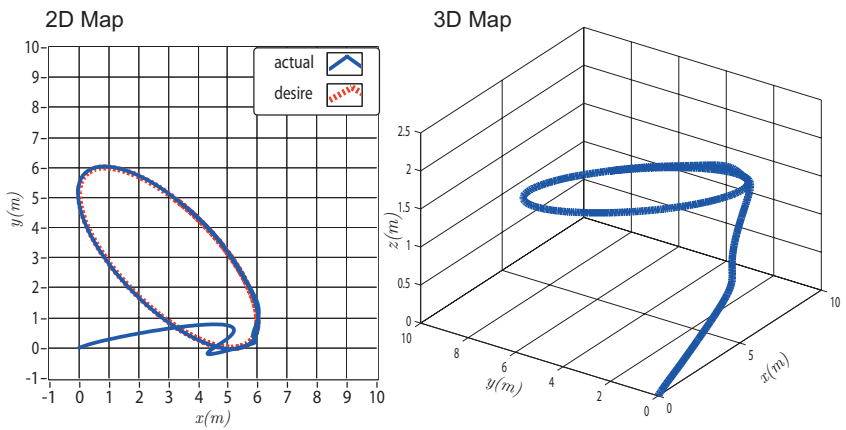

Fig. 15. Simulation result of ellipse path tracking. (a) The tracking performance in $2 \mathrm{~d}$. (b) The tracking performance in $3 \mathrm{~d}$.

\subsection{Figure-Eight Path Tracking :}

The task was climbing to $2 \mathrm{~m}$ from the ground and then follow the figure eight Path. The path described as : $x_{d}(t)=3+3 \sin (0.2 \pi t)$ and $y_{d}(t)=3+3 \cos (0.1 \pi t)$. Fig. 16 (a),(b) show the tracking response in $y$ and $x$ directions. Fig. 17](a),(b) show the attitude response. Fig. 18. (a) shows the altitude response. Fig. 18 (b) shows the yaw response. Fig. 19 (a),(b) show the figure eight tracking performance of the quad in 2d-plan and 3d-plan. 

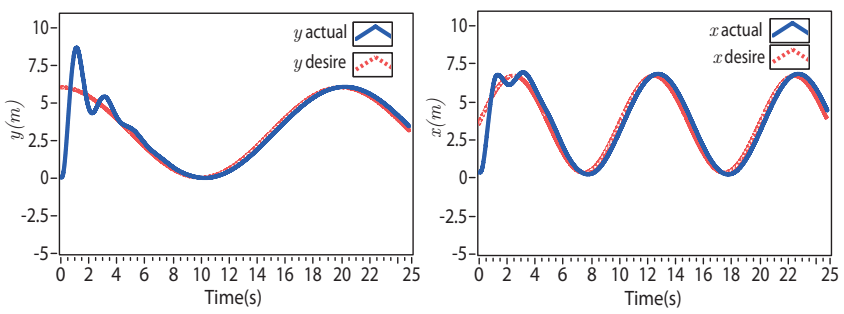

Fig. 16. The position signals generated to track the figure eight trajectory. (a) The desired and the actual values of $y$. (b) The desired and the actual values of $x$.
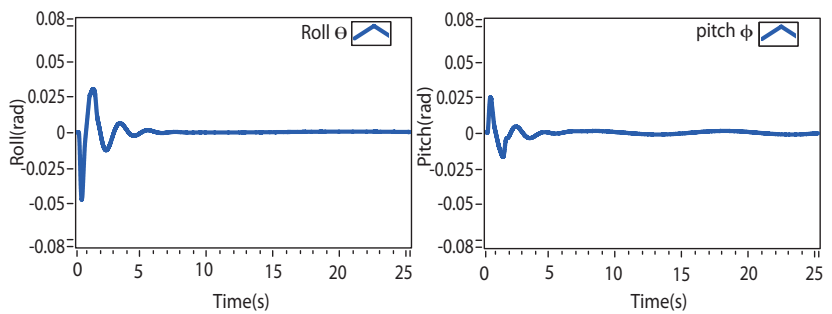

Fig. 17. The attitude signals generated to track the figure eight trajectory. (a) the roll response. (b) the pitch response.
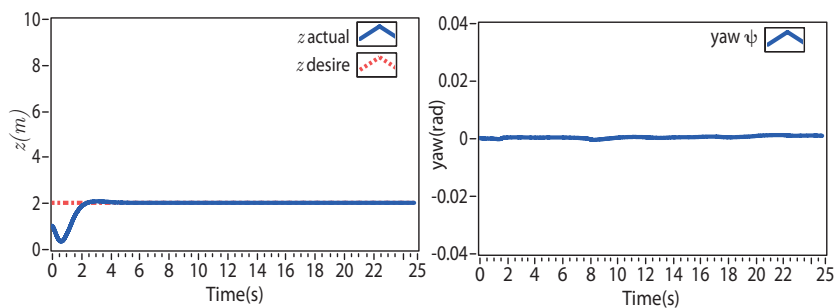

Fig. 18. (a) the altitude response. (b) the yaw response.
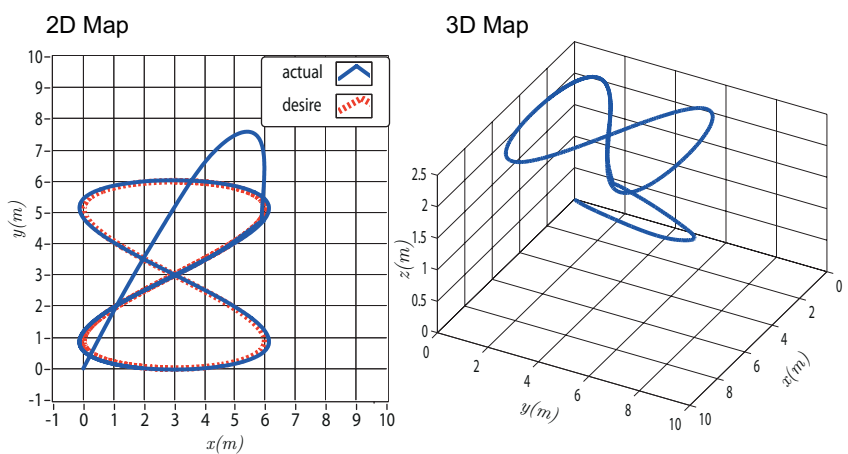

Fig. 19. Simulation result of figure eight path tracking. (a) The tracking performance in $2 \mathrm{~d}$. (b) The tracking performance in $3 \mathrm{~d}$.

\section{CONCLUSIONS}

In this paper a real time trajectory tracking based on state feedback with integral action control scheme is developed for a quadrotor. In order to test the performance of the designed controller, the kinematic and dynamic models of quadrotor are included to implement simulation in NI-LabVIEW. The simulation results for several scenarios show that all the state variables converge to their reference values. To validate the proposed controller practically, several experiments are under development in our lab.

\section{REFERENCES}

[1] M. Tarhan and E. Altug, "EKF based attitude estimation and stabilization of a Quadrotor UAV using vanishing points in catadioptric images," Trans. of Intelligent and Robotic Systems, Vol. 62, pp. 587607, 2011.

[2] T. Tomic, K. Schmid, P. Lutz, A. Domel, M. Kassecker, E. Mair, I. L.Grixa, F. Ruess, M. Suppa, and D. Burschka, "Toward a fully autonomous UAV: research platform for indoor and outdoor urban search and rescue," Trans. of Robotics and Automation Magazine, Vol. 19, pp. 4656, 2012.

[3] P.E.I. Pounds, D.R. Bersak, and A.M. Dollar, "Grasping from the air: Hovering capture and load stability," Proc. of the 2011 Int. Conf. on Robotics and Automation (ICRA), pp. 24912498, 2011.

[4] Zuo Z., Ru P, "Augmented L1 Adaptive Tracking Control of Quad-Rotor Unmanned Aircrafts," IEEE Transactions on Aerospace and Electronic Systems, Vol. 50, pp. 3090-3101, 2014.

[5] Raffo, G.V., Ortega, M.G., Rubio, F.R, "An integral predictive/nonlinear H8 control structure for a quadrotor helicopter," Automatica, Vol. 46(1), 2010.

[6] B. Erginer, E. Altug, "Modeling and PD control of a quadrotor VTOL vehicle," Intelligent Vehicles Symposium, Vol. 6, pp. 894-899, 2007.

[7] H. Bolandi, M. Rezaei , "Nemati H.Attitude Control of a quadrotor with Optimized PID," Intelligent Control and Automation, Vol. 8, pp. 335-342, 2013.

[8] S. Bouabdallah, "PID vs LQ Control Techniques Applied to an Indoor Micro quadrotor" IEEE/RS.J Internationel Conference On Intelligent Robots and Systems, Vol. 10, pp. 2451-2456, 2004.

[9] Basri, M.A.M., Husain, A.R., Danapalasingam, K.A, "Trajectory tracking control design with command-filtered compensation for a quadrotor," Sadhana, Vol. 40(5), pp. 1531-1553, 2015.

[10] Lee, D., Kim, H.J., Sastry, S, "Feedback linearization vs. adaptive sliding mode control for a quadrotor helicopter," International Journal of control, Automation and systems, Vol. 7(3), pp. 419-428, 2009.

[11] Besnarda, L., Shtesselb, Y.B., Landrum, B, "Adaptive tracking control of underactuated quadrotor unmanned aerial vehicles via backstepping," American Control Conference (ACC)), Vol. 349(2), pp. 658-684, 2012.

[12] Zuo, Z, "Stabilization and trajectory tracking control for underactuated quadrotor helicopter subject to wind-gust disturbance," IET control theory and applications, Vol. 4(11), pp. 2343, 2010.

[13] Castillo, P., Dzul, A., Lozano, R, "Real-time stabilization and tracking of a four rotor mini-rotorcraft," European Control Conference (ECC), Vol. 20, pp. 3123-3128, 2003.

[14] Huang, M., Xian, B., Diao, C., Yang, K., Feng, Y, "Quadrotor vehicle control via sliding mode controller driven by sliding mode disturbance observer," Journal of the Franklin Institute, pp. 20762081, 2010.

[15] Lee, D., Nataraj, C., Burg, T.C., Dawson, D.M, "Adaptive tracking control of an underactuated aerial vehicle," American Control Conference (ACC)), pp. 23262331, 2011. 
[16] S. Bouabdallah, "Design and Control of Quadrotors with Application to Autonomous Flying" PhD Thesis, Lausanne Polytechnic University, Lausanne,Switzerland, 2007.

[17] Lee. Daewon, H. Jin, Shankar Sastry, "Feedback linearization vs adaptive sliding mode control for a quadrotor helicopter," 44th International Journal of Control,Automation and Systems, Vol. 7, pp. 419-428, 2009.

[18] A. Ryan and J.K.Hedrick, "A mode-switching path planner for UAV-assisted search and rescue," 44th IEEE Conference, Vol. 12, pp. 1471-1476, 15 December 2005.

[19] A. L. Salih , M. Moghavvemil, H. A. F. Mohamed and K. S. Gaeid,""Flight PID Controller Design for a UAV Quadcopter,'Scientific Research and Essays, Vol. 5,NO.23, pp. 3660-3667, 2010.

[20] A. Z. Azfar and D. Hazry, "Simple GUI Design for Moni- toring of a Remotely Operated Quadcopter Unmanned Aerial vehicle,"Processing of the 7th International Colloquium on Signal Processing and its Applications (CSPA), pp. 23-27, 6 Mach 2011.

[21] P. Hippe and J. Deutscher ,"Design of Observer-based Compensators,"'ch.State Feedback Control,"Springer London, pp. $17-26,2009$. 